\title{
A Suzuki Type Coupled Fixed Point Theorem for Generalized Multivalued Mapping
}

\author{
Pushpendra Semwal and Ramesh Chandra Dimri \\ Department of Mathematics, HNB Garhwal University, Srinagar Garhwal, Pauri Garhwal, Uttarakhand 246174, India \\ Correspondence should be addressed to Pushpendra Semwal; psrsdm@gmail.com \\ Received 4 November 2013; Accepted 24 January 2014; Published 15 April 2014 \\ Academic Editor: Mohamed Amine Khamsi
}

Copyright (C) 2014 P. Semwal and R. C. Dimri. This is an open access article distributed under the Creative Commons Attribution License, which permits unrestricted use, distribution, and reproduction in any medium, provided the original work is properly cited.

We obtain a new Suzuki type coupled fixed point theorem for a multivalued mapping $T$ from $X \times X$ into $\mathrm{CB}(X)$, satisfying a generalized contraction condition in a complete metric space. Our result unifies and generalizes various known comparable results in the literature. We also give an application to certain functional equations arising in dynamic programming.

\section{Introduction and Preliminaries}

In 2008, Suzuki [1] introduced a new type of mappings which generalize the well-known Banach contraction principle [2], and, further, Kikkawa and Suzuki [3] proved a Kannan [4] version of mappings.

Theorem 1 (see [3]). Let $(X, d)$ be a complete metric space. Let $T: X \rightarrow X$ be a self-map and let $\phi:[0,1) \rightarrow(1 / 2,1]$ be defined by

$$
\phi(r)= \begin{cases}1 & \text { if } 0 \leq r \leq \frac{1}{\sqrt{2}} \\ \frac{1}{1+r} & \text { if } \frac{1}{\sqrt{2}} \leq r \leq 1\end{cases}
$$

Let $\alpha \in[0,1 / 2)$ and $r=\alpha /(1-\alpha) \in[0,1)$. Suppose that

$$
\begin{aligned}
\phi(r) d(x, T x) & \leq d(x, y) \text { implies } \\
d(T x, T y) & \leq \alpha(d(x, T x)+d(y, T y))
\end{aligned}
$$

for all $x, y \in X$. Then, $T$ has a unique fixed point $z$, and $\lim _{n} T^{n} x=z$ holds for every $x \in X$.
Let $(X, d)$ be a metric space. We denote by $\mathrm{CB}(X)$ the family of all nonempty, closed bounded subsets of $X$. Let $H$ be a Hausdorff metric; that is,

$$
H(A, B)=\max \left\{\sup _{a \in A} d(a, B), \sup _{b \in B} d(A, b)\right\}
$$

for $A, B \in \mathrm{CB}(X)$, where $d(x, B)=\inf _{y \in B} d(x, y)$.

Nadler [5] proved multivalued extension of the Banach contraction principle as follows.

Let $(X, d)$ be a complete metric space and let $T$ be a mapping from $X$ into $\mathrm{CB}(X)$. Assume that there exists $r \in$ $[0,1)$ such that

$$
H(T x, T y) \leq r d(x, y)
$$

for all $x, y \in X$. Then, there exists $z \in X$ such that $z \in T z$. Many fixed point theorems have been proved by various authors as a generalization of Nadler's theorem [69]. One of the general fixed point theorems for a generalized multivalued mapping appears in [10]. 
Theorem 2 (see $[11])$. Let $(X, d)$ be a complete metric space and let $T$ be a mapping from $X$ into $\mathrm{CB}(X)$. Assume that there exists a function $\phi$ from $[0,1)$ into $(0,1]$ defined by

$$
\phi(r)= \begin{cases}1 & \text { if } 0 \leq r<\frac{1}{2}, \\ 1-r & \text { if } \frac{1}{2} \leq r<1,\end{cases}
$$

such that

$$
\begin{gathered}
\phi(r) d(x, T x) \leq d(x, y) \text { implies } \\
H(T x, T y) \leq r \max \{d(x, y), d(x, T x), d(y, T y), \\
\left.\frac{d(x, T y)+d(y, T x)}{2}\right\}
\end{gathered}
$$

for all $x, y \in X$. Then, there exists $z \in X$ such that $z \in T z$.

Bhaskar and Lakshmikantham [12] introduced the concept of coupled fixed point for a mapping $F$ from $X \times X$ to $X$ and established some coupled fixed point theorems in partially ordered sets. As an application, they studied the existence and uniqueness of solution for a periodic boundary value problem associated with a first order ordinary differential equation.

Definition 3. Let $(X, d)$ be a metric space and $F: X \times X \rightarrow$ $2^{X}$. An element $(x, y) \in X \times X$ is called a coupled fixed point of $F$ if $x \in F(x, y)$ and $y \in F(y, x)$.

The aim of this paper is to obtain coupled fixed point for a multivalued mapping $T: X \times X \rightarrow \mathrm{CB}(X)$ which satisfies the generalized contraction condition in complete metric spaces. Our results unify, extend, and generalize various known comparable results in the literature.

\section{Main Results}

Now, we shall prove our main result.

Firstly, we define a nonincreasing function $\phi$ from $[0,1)$ into $[0,1)$ by

$$
\phi(r)= \begin{cases}r & \text { if } 0 \leq r<\frac{1}{2}, \\ 1-r & \text { if } \frac{1}{2} \leq r<1 .\end{cases}
$$

Theorem 4. Let $(X, d)$ be a complete metric space and let $T$ be a mapping from $X \times X$ into $\mathrm{CB}(X)$. Assume that there exists $r \in[0,1)$ such that

$$
\phi(r)[d(x, T(x, y))+d(u, T(u, v))] \leq d(x, u)+d(y, v)
$$

implies

$$
\begin{aligned}
& H(T(x, y), T(u, v)) \\
& \leq \frac{r}{2} \max \{d(x, u)+d(y, v), d(x, T(x, y)) \\
&+ d(y, T(y, x)), d(u, T(u, v))+d(v, T(v, u)), \\
& \frac{d(x, T(u, v))+d(y, T(v, u))}{2}, \\
&\left.\frac{d(u, T(x, y))+d(v, T(y, x))}{2}\right\}
\end{aligned}
$$

for all $x, y, u, v \in X$. Then, there exist $z, z^{\prime} \in X$ such that $z \in T\left(z, z^{\prime}\right)$ and $z^{\prime} \in T\left(z^{\prime}, z\right)$.

Proof. Let $r_{1}$ be a real number such that $0 \leq r<r_{1}<1$, and $x_{1}, y_{1} \in X$ such that $x_{2} \in T\left(x_{1}, y_{1}\right)$ and $y_{2} \in T\left(y_{1}, x_{1}\right)$. Since $x_{2} \in T\left(x_{1}, y_{1}\right)$ and $y_{2} \in T\left(y_{1}, x_{1}\right)$, then

$$
\begin{aligned}
& d\left(x_{2}, T\left(x_{2}, y_{2}\right)\right) \leq H\left(T\left(x_{1}, y_{1}\right), T\left(x_{2}, y_{2}\right)\right), \\
& d\left(y_{2}, T\left(y_{2}, x_{2}\right)\right) \leq H\left(T\left(y_{1}, x_{1}\right), T\left(y_{2}, x_{2}\right)\right),
\end{aligned}
$$

and, as $\phi(r)<1$,

$$
\begin{gathered}
\phi(r)\left(d\left(x_{2}, T\left(x_{2}, y_{2}\right)\right)+d\left(y_{2}, T\left(y_{2}, x_{2}\right)\right)\right) \\
\leq d\left(x_{2}, T\left(x_{2}, y_{2}\right)\right)+d\left(y_{2}, T\left(y_{2}, x_{2}\right)\right) \\
\leq d\left(x_{2}, x_{3}\right)+d\left(y_{2}, y_{3}\right) .
\end{gathered}
$$

Thus, from assumption (10), we have

$$
\begin{aligned}
& d\left(x_{2}, T\left(x_{2}, y_{2}\right)\right) \\
& \leq H\left(T\left(x_{1}, y_{1}\right), T\left(x_{2}, y_{2}\right)\right) \\
& \leq \frac{r}{2} \max \left\{d\left(x_{1}, x_{2}\right)+d\left(y_{1}, y_{2}\right), d\left(x_{1}, T\left(x_{1}, y_{1}\right)\right)\right. \\
&+ d\left(y_{1}, T\left(y_{1}, x_{1}\right)\right), d\left(x_{2}, T\left(x, y_{2}\right)\right) \\
&+ d\left(y_{2}, T\left(y_{2}, x_{2}\right)\right), \\
& \frac{d\left(x_{1}, T\left(x_{2}, y_{2}\right)\right)+d\left(y_{1}, T\left(y_{2}, x_{2}\right)\right)}{2}, \\
&\left.\frac{d\left(x_{2}, T\left(x_{1}, y_{1}\right)\right)+d\left(y_{2}, T\left(y_{1}, x_{1}\right)\right)}{2}\right\},
\end{aligned}
$$




$$
\begin{aligned}
& d\left(x_{2}, x_{3}\right) \\
& \leq \frac{r}{2} \max \left\{d\left(x_{1}, x_{2}\right)+d\left(y_{1}, y_{2}\right), d\left(x_{1}, x_{2}\right)\right. \\
& +d\left(y_{1}, y_{2}\right), d\left(x_{2}, x_{3}\right)+d\left(y_{2}, y_{3}\right), \\
& \quad \frac{d\left(x_{1}, x_{3}\right)+d\left(y_{1}, y_{3}\right),}{2}, \\
& \left.\frac{d\left(x_{2}, x_{2}\right)+d\left(y_{2}, y_{2}\right)}{2}\right\}, \\
& d\left(y_{2}, T\left(y_{2}, x_{2}\right)\right) \\
& \leq H\left(T\left(y_{1}, x_{1}\right), T\left(y_{2}, x_{2}\right)\right) \\
& \leq \frac{r}{2} \max \left\{d\left(x_{1}, x_{2}\right)+d\left(y_{1}, y_{2}\right), d\left(x_{1}, T\left(x_{1}, y_{1}\right)\right)\right. \\
& +d\left(y_{1}, T\left(y_{1}, x_{1}\right)\right), d\left(x_{2}, T\left(x, y_{2}\right)\right) \\
& +d\left(y_{2}, T\left(y_{2}, x_{2}\right)\right), \\
& \frac{d\left(x_{1}, T\left(x_{2}, y_{2}\right)\right)+d\left(y_{1}, T\left(y_{2}, x_{2}\right)\right)}{2}, \\
& \left.\frac{d\left(x_{2}, T\left(x_{1}, y_{1}\right)\right)+d\left(y_{2}, T\left(y_{1}, x_{1}\right)\right)}{2}\right\},
\end{aligned}
$$

$d\left(y_{2}, y_{3}\right)$

$$
\begin{aligned}
\leq \frac{r}{2} \max & \left\{d\left(x_{1}, x_{2}\right)+d\left(y_{1}, y_{2}\right), d\left(x_{1}, x_{2}\right)\right. \\
& +d\left(y_{1}, y_{2}\right), d\left(x_{2}, x_{3}\right)+d\left(y_{2}, y_{3}\right), \\
& \frac{d\left(x_{1}, x_{3}\right)+d\left(y_{1}, y_{3}\right)}{2}, \\
& \left.\frac{d\left(x_{2}, x_{2}\right)+d\left(y_{2}, y_{2}\right)}{2}\right\} .
\end{aligned}
$$

Adding (14) and (16), we have

$$
\begin{gathered}
d\left(x_{2}, x_{3}\right)+d\left(y_{2}, y_{3}\right) \\
\leq r \max \left\{d\left(x_{1}, x_{2}\right)+d\left(y_{1}, y_{2}\right), d\left(x_{2}, x_{3}\right)+d\left(y_{2}, y_{3}\right),\right. \\
\left.\frac{d\left(x_{1}, x_{3}\right)+d\left(y_{1}, y_{3}\right)}{2}\right\} .
\end{gathered}
$$

If $\max \left\{d\left(x_{1}, x_{2}\right)+d\left(y_{1}, y_{2}\right), d\left(x_{2}, x_{3}\right)+d\left(y_{2}, y_{3}\right)\right.$, $\left.\left(d\left(x_{1}, x_{3}\right)+d\left(y_{1}, y_{3}\right)\right) / 2\right\}=d\left(x_{2}, x_{3}\right)+d\left(y_{2}, y_{3}\right)$, then we have $d\left(x_{2}, x_{3}\right)+d\left(y_{2}, y_{3}\right) \leq r\left(d\left(x_{2}, x_{3}\right)+d\left(y_{2}, y_{3}\right)\right)$ as $r<1$; a contradiction. Therefore,

$$
\begin{aligned}
& d\left(x_{2}, x_{3}\right)+d\left(y_{2}, y_{3}\right) \\
& \leq r \max \left\{d\left(x_{1}, x_{2}\right)+d\left(y_{1}, y_{2}\right), \frac{d\left(x_{1}, x_{3}\right)+d\left(y_{1}, y_{3}\right)}{2}\right\} .
\end{aligned}
$$

Again, if $\max \left\{d\left(x_{1}, x_{2}\right)+d\left(y_{1}, y_{2}\right),\left(d\left(x_{1}, x_{3}\right)+d\left(y_{1}\right.\right.\right.$, $\left.\left.\left.y_{3}\right)\right) / 2\right\}=d\left(x_{1}, x_{2}\right)+d\left(y_{1}, y_{2}\right)$, we get

$$
d\left(x_{2}, x_{3}\right)+d\left(y_{2}, y_{3}\right) \leq r\left(d\left(x_{1}, x_{2}\right)+d\left(y_{1}, y_{2}\right)\right)
$$

and if $\max \left\{d\left(x_{1}, x_{2}\right)+d\left(y_{1}, y_{2}\right),\left(d\left(x_{1}, x_{3}\right)+d\left(y_{1}, y_{3}\right)\right) / 2\right\}=$ $\left(d\left(x_{1}, x_{3}\right)+d\left(y_{1}, y_{3}\right)\right) / 2$, we get

$$
d\left(x_{2}, x_{3}\right)+d\left(y_{2}, y_{3}\right) \leq r \frac{d\left(x_{1}, x_{3}\right)+d\left(y_{1}, y_{3}\right)}{2} .
$$

Using triangle inequality, we obtain

$$
\begin{aligned}
& d\left(x_{2}, x_{3}\right)+d\left(y_{2}, y_{3}\right) \\
& \quad \leq r \frac{d\left(x_{1}, x_{2}\right)+d\left(x_{2}, x_{3}\right)+d\left(y_{1}, y_{2}\right)+d\left(y_{2}, y_{3}\right)}{2} .
\end{aligned}
$$

This implies

$$
d\left(x_{2}, x_{3}\right)+d\left(y_{2}, y_{3}\right) \leq\left(\frac{r}{2-r}\right) d\left(x_{1}, x_{2}\right)+d\left(y_{1}, y_{2}\right) .
$$

Hence, there exist $x_{3}, y_{3} \in X$ with $x_{3} \in T\left(x_{2}, y_{2}\right)$ and $y_{3} \in T\left(y_{2}, x_{2}\right)$ such that

$$
\begin{aligned}
& d\left(x_{2}, x_{3}\right)+d\left(y_{2}, y_{3}\right) \leq r\left(d\left(x_{1}, x_{2}\right)+d\left(y_{1}, y_{2}\right)\right), \\
& d\left(x_{2}, x_{3}\right)+d\left(y_{2}, y_{3}\right) \leq\left(\frac{r}{2-r}\right) d\left(x_{1}, x_{2}\right)+d\left(y_{1}, y_{2}\right) .
\end{aligned}
$$

Thus, we construct such sequences $\left\{x_{n}\right\}$ and $\left\{y_{n}\right\}$ in $X$ such that $x_{n+1} \in T\left(x_{n}, y_{n}\right), y_{n+1} \in T\left(y_{n}, x_{n}\right)$, and

$$
\begin{gathered}
d\left(x_{n}, x_{n+1}\right)+d\left(y_{n}, y_{n+1}\right) \leq r\left(d\left(x_{n-1}, x_{n}\right)+d\left(y_{n-1}, y_{n}\right)\right), \\
d\left(x_{n}, x_{n+1}\right)+d\left(y_{n}, y_{n+1}\right) \\
\leq\left(\frac{r}{2-r}\right) d\left(x_{n-1}, x_{n}\right)+d\left(y_{n-1}, y_{n}\right) .
\end{gathered}
$$

Then, we have

$$
\begin{aligned}
& \sum_{n=1}^{\infty}\left(d\left(x_{n}, x_{n+1}\right)+d\left(y_{n}, y_{n+1}\right)\right) \\
& \quad \leq \sum_{n=1}^{\infty} r^{n}\left(d\left(x_{1}, x_{2}\right)+d\left(y_{1}, y_{2}\right)\right)<\infty, \\
& \sum_{n=1}^{\infty}\left(d\left(x_{n}, x_{n+1}\right)+d\left(y_{n}, y_{n+1}\right)\right) \\
& \quad \leq \sum_{n=1}^{\infty}\left(\frac{r}{2-r}\right)^{n}\left(d\left(x_{1}, x_{2}\right)+d\left(y_{1}, y_{2}\right)\right)<\infty .
\end{aligned}
$$


Hence, we conclude that in both cases the sequences $\left\{x_{n}\right\}$ and $\left\{y_{n}\right\}$ are Cauchy sequences. Since $X$ is complete, there are some points $z$ and $z^{\prime}$ in $X$ such that $\lim _{n \rightarrow \infty} x_{n}=z$ and $\lim _{n \rightarrow \infty} y_{n}=z^{\prime}$.

Now, we shall show that

$$
\begin{aligned}
& d(z, T(x, y))+d\left(z^{\prime}, T(y, x)\right) \\
& \leq r \max \{\left\{(z, x)+d\left(z^{\prime}, y\right),\right. \\
&d(x, T(x, y))+d(y, T(y, x))\}
\end{aligned}
$$

for all $x \in X /\{z\}$ and $y \in X /\left\{z^{\prime}\right\}$. Since $x_{n} \rightarrow x$ and $y_{n} \rightarrow y$, there exists $n_{0} \in N$ such that $d\left(z, x_{n}\right)+d\left(z^{\prime}, y_{n}\right) \leq$ $(1 / 3)\left(d(z, x)+d\left(z^{\prime}, y\right)\right)$ for all $n \geq n_{0}$. Therefore

$$
\begin{aligned}
\phi(r) & d\left(x_{n}, T\left(x_{n}, y_{n}\right)\right)+d\left(y_{n}, T\left(y_{n}, x_{n}\right)\right) \\
& \leq r\left(d\left(x_{n+1}, x_{n}\right)+d\left(y_{n+1}, y_{n}\right)\right) \\
& \leq d\left(x_{n}, z\right)+d\left(z, x_{n+1}\right)+d\left(y_{n}, z^{\prime}\right)+d\left(z^{\prime}, y_{n+1}\right) \\
& \leq d\left(x_{n}, z\right)+d\left(y_{n}, z^{\prime}\right)+d\left(z, x_{n+1}\right)+d\left(z^{\prime}, y_{n+1}\right) \\
& \leq \frac{2}{3}\left(d(x, z)+d\left(y, z^{\prime}\right)\right) .
\end{aligned}
$$

Thus,

$$
\begin{gathered}
\phi(r) d\left(x_{n}, T\left(x_{n}, y_{n}\right)\right)+d\left(y_{n}, T\left(y_{n}, x_{n}\right)\right) \\
\leq \frac{2}{3}\left(d(x, z)+d\left(y, z^{\prime}\right)\right) .
\end{gathered}
$$

$$
\begin{aligned}
& \text { Since } \\
& \begin{aligned}
\frac{2}{3}(d & \left.(x, z)+d\left(y, z^{\prime}\right)\right) \\
& =\left(d(x, z)+d\left(y, z^{\prime}\right)\right)-\frac{1}{3}\left(d(x, z)+d\left(y, z^{\prime}\right)\right) \\
& \leq\left(d(x, z)+d\left(y, z^{\prime}\right)\right)-\left(d\left(x_{n}, z\right)+d\left(y_{n}, z^{\prime}\right)\right) \\
& \leq d\left(x_{n}, x\right)+d\left(y_{n}, y\right),
\end{aligned}
\end{aligned}
$$

from (29) we have

$$
\begin{aligned}
& \phi(r) d\left(x_{n}, T\left(x_{n}, y_{n}\right)\right)+d\left(y_{n}, T\left(y_{n}, x_{n}\right)\right) \\
& \leq d\left(x_{n}, x\right)+d\left(y_{n}, y\right) .
\end{aligned}
$$

Then, from (10) we have

$$
\begin{aligned}
& H\left(T\left(x_{n}, y_{n}\right), T(x, y)\right) \\
& \leq \frac{r}{2} \max \{\left\{d\left(x_{n}, x\right)+d\left(y_{n}, y\right), d\left(x_{n}, T\left(x_{n}, y_{n}\right)\right)\right. \\
&+d\left(y_{n}, T\left(y_{n}, x_{n}\right)\right), d(x, T(x, y)) \\
&+d(y, T(y, x)), \\
& \frac{d\left(x_{n}, T(x, y)\right)+d\left(y_{n}, T(y, x)\right)}{2}, \\
&\left.\frac{d\left(x, T\left(x_{n}, y_{n}\right)\right)+d\left(y, T\left(y_{n}, x_{n}\right)\right)}{2}\right\},
\end{aligned}
$$

$$
\begin{aligned}
& H\left(T\left(y_{n}, x_{n}\right), T(y, x)\right) \\
& \leq \frac{r}{2} \max \{ d\left(x_{n}, x\right)+d\left(y_{n}, y\right), d\left(x_{n}, T\left(x_{n}, y_{n}\right)\right) \\
&+d\left(y_{n}, T\left(y_{n}, x_{n}\right)\right), d(x, T(x, y)) \\
&+d(y, T(y, x)), \\
& \frac{d\left(x_{n}, T(x, y)\right)+d\left(y_{n}, T(y, x)\right)}{2}, \\
&\left.\frac{d\left(x, T\left(x_{n}, y_{n}\right)\right)+d\left(y, T\left(y_{n}, x_{n}\right)\right)}{2}\right\} .
\end{aligned}
$$

By adding, (32) and (33), we get

$$
\begin{aligned}
& d\left(x_{n+1}, T(x, y)\right)+d\left(y_{n+1}, T(y, x)\right) \\
& \leq r \max \{\left\{d\left(x_{n}, x\right)+d\left(y_{n}, y\right), d\left(x_{n}, x_{n+1}\right)\right. \\
&+d\left(y_{n}, y_{n+1}\right), d(x, T(x, y)) \\
&+d(y, T(y, x)), \\
& \frac{d\left(x_{n}, T(x, y)\right)+d\left(y_{n}, T(y, x)\right)}{2}, \\
&\left.\frac{d\left(x, x_{n+1}\right)+d\left(y, y_{n+1}\right)}{2}\right\} .
\end{aligned}
$$

Letting $n$ tends to $\infty$, we obtain

$$
\begin{aligned}
d(z, T(x, y))+d\left(z^{\prime}, T(y, x)\right) \\
\leq r \max \left\{d(z, x)+d\left(z^{\prime}, y\right), d(x, T(x, y))\right. \\
+d(y, T(y, x)), \\
\left.\quad \frac{d(z, T(x, y))+d\left(z^{\prime}, T(y, x)\right)}{2}\right\} .
\end{aligned}
$$


Hence, we have:

$$
\begin{gathered}
d(z, T(x, y))+d\left(z^{\prime}, T(y, x)\right) \\
\leq r \max \left\{d(z, x)+d\left(z^{\prime}, y\right), d(x, T(x, y))\right. \\
+d(y, T(y, x))\} .
\end{gathered}
$$

Now, we shall prove that $z \in T\left(z, z^{\prime}\right)$ and $z^{\prime} \in T\left(z^{\prime}, z\right)$.

Case 1. First, we consider the case $0 \leq r<1 / 2$. Suppose, on contrary, that $z \notin T\left(z, z^{\prime}\right)$ and $z^{\prime} \notin\left(z^{\prime}, z\right)$. Let $a \in$ $T\left(z, z^{\prime}\right)$ and $b \in T\left(z^{\prime}, z\right)$ be such that $2 r\left\{d(a, z)+d\left(b, z^{\prime}\right)\right\}<$ $d\left(z, T\left(z, z^{\prime}\right)\right)+d\left(z^{\prime}, T\left(z^{\prime}, z\right)\right)$. Since $a \in T\left(z, z^{\prime}\right)$ and $b \in$ $T\left(z^{\prime}, z\right)$ imply $a \neq z$ and $b \neq z^{\prime}$, from (10), we have

$$
\begin{aligned}
& d(z, T(a, b))+d\left(z^{\prime}, T(b, a)\right) \\
& \leq r \max \left\{d(z, a)+d\left(z^{\prime}, b\right), d(a, T(a, b))+d(b, T(b, a))\right\} .
\end{aligned}
$$

On the other hand, since $\phi(r) d\left(z, T\left(z, z^{\prime}\right)\right)+d\left(z^{\prime}, T\left(z^{\prime}\right.\right.$, $z)) \leq d\left(z, T\left(z, z^{\prime}\right)\right)+d\left(z^{\prime}, T\left(z^{\prime}, z\right)\right)<d(z, a)+d\left(z^{\prime}, b\right)$, from (10), we get

$$
\begin{aligned}
& H\left(T\left(z, z^{\prime}\right), T(a, b)\right) \\
& \leq \frac{r}{2} \max \left\{d(z, a)+d\left(z^{\prime}, b\right), d\left(z, T\left(z, z^{\prime}\right)\right)\right. \\
& +d\left(z^{\prime}, T\left(z^{\prime}, z\right)\right), d(a, T(a, b)) \\
& +d(b, T(b, a)), \\
& \frac{d(z, T(a, b))+d\left(z^{\prime}, T(b, a)\right)}{2}, \\
& \left.\frac{d\left(a, T\left(z, z^{\prime}\right)\right)+d\left(b, T\left(z^{\prime}, z\right)\right)}{2}\right\} \text {, } \\
& H\left(T\left(z^{\prime}, z\right), T(b, a)\right) \\
& \leq \frac{r}{2} \max \left\{d(z, a)+d\left(z^{\prime}, b\right), d\left(z, T\left(z, z^{\prime}\right)\right)\right. \\
& +d\left(z^{\prime}, T\left(z^{\prime}, z\right)\right), d(a, T(a, b)) \\
& +d(b, T(b, a)), \\
& \frac{d(z, T(a, b))+d\left(z^{\prime}, T(b, a)\right)}{2}, \\
& \left.\frac{d\left(a, T\left(z, z^{\prime}\right)\right)+d\left(b, T\left(z^{\prime}, z\right)\right)}{2}\right\} \text {. }
\end{aligned}
$$

By adding, (38) and (39), we have

$$
\begin{aligned}
d(a, T(a, b))+ & d(b, T(b, a)) \\
\leq r \max & \left\{d(z, a)+d\left(z^{\prime}, b\right), d(a, T(a, b))\right. \\
& +d(b, T(b, a)), \\
& \left.\frac{d(z, T(a, b))+d\left(z^{\prime}, T(b, a)\right)}{2}\right\} .
\end{aligned}
$$

This implies

$d(a, T(a, b))+d(b, T(b, a))$

$$
\leq r\left\{d(z, a)+d\left(z^{\prime}, b\right)\right\}<d(z, a)+d\left(z^{\prime}, b\right) .
$$

And, from (40), we have

$d(z, T(a, b))+d\left(z^{\prime}, T(b, a)\right) \leq r\left\{d(z, a)+d\left(z^{\prime}, b\right)\right\}$.

Therefore, we obtain

$$
\begin{aligned}
& d\left(z, T\left(z, z^{\prime}\right)\right)+d\left(z^{\prime}, T\left(z^{\prime}, z\right)\right) \\
& \leq d(z, T(a, b))+H\left(T(a, b), T\left(z, z^{\prime}\right)\right) \\
&+d\left(z^{\prime}, T(b, a)\right)+H\left(T(b, a), T\left(z^{\prime}, z\right)\right) \\
& \leq 2 r\left(d(z, a)+d\left(z^{\prime}, b\right)\right) \\
&< d\left(z, T\left(z, z^{\prime}\right)\right)+d\left(z^{\prime}, T\left(z^{\prime}, z\right)\right) .
\end{aligned}
$$

This is a contradiction. As a result, we have $z \in T\left(z, z^{\prime}\right)$ and $z^{\prime} \in T\left(z^{\prime}, z\right)$.

Case 2. Now, we consider the case $1 / 2 \leq r<1$. We shall first prove that

$$
\begin{aligned}
H(T(x, y), T( & \left.\left(z, z^{\prime}\right)\right)+H\left(T(y, x), T\left(z^{\prime}, z\right)\right) \\
\leq r \max & \left\{d(x, z)+d\left(y, z^{\prime}\right), d(x, T(x, y))\right. \\
& +d(y, T(y, x)), d\left(z, T\left(z, z^{\prime}\right)\right) \\
& +d\left(z^{\prime}, T\left(z^{\prime}, z\right)\right), \\
& \frac{d\left(x, T\left(z, z^{\prime}\right)\right)+d\left(y, T\left(z^{\prime}, z\right)\right)}{2} \\
& \left.\frac{d(z, T(x, y))+d\left(z^{\prime}, T(y, x)\right)}{2}\right\}
\end{aligned}
$$

for all $x, y \in X$. If $x=z$ and $y=z^{\prime}$, then the previous equation (44) obviously holds. Hence, let us assume $x \neq z$ 
and $y \neq z^{\prime}$. Then, for every $n \in N$, there exist sequences $t_{n} \in T(x, y)$ and $t_{n}^{\prime} \in T(y, x)$ such that

$$
\begin{aligned}
d\left(z, t_{n}\right) & +d\left(z^{\prime}, t_{n}^{\prime}\right) \\
\leq & r\left\{d(z, T(x, y))+d\left(z^{\prime}, T(y, x)\right)\right\} \\
& +\frac{1}{n} d(z, x)+d\left(z^{\prime}, y\right) .
\end{aligned}
$$

Then for all $n \in N$, we have

$$
\begin{aligned}
& d(x, T(x, y))+d(y, T(y, x)) \\
& \leq d\left(x, t_{n}\right)+d\left(y, t_{n}^{\prime}\right) \\
& \leq d(x, z)+d\left(z, t_{n}\right)+d\left(y, z^{\prime}\right)+d\left(z^{\prime}, t_{n}^{\prime}\right) \\
& \leq d(x, z)+d\left(y, z^{\prime}\right)+d(z, T(x, y))+d\left(z^{\prime}, T(y, x)\right) \\
& \quad+\frac{1}{n}\left(d(x, z)+d\left(y, z^{\prime}\right)\right) \\
& \leq d(x, z)+d\left(y, z^{\prime}\right) \\
& \quad+r \max \left\{d(x, z)+d\left(y, z^{\prime}\right), d(x, T(x, y))\right. \\
& \quad+d(y, T(y, x))\}+\frac{1}{n}\left(d(x, z)+d\left(y, z^{\prime}\right)\right) .
\end{aligned}
$$
we get

If $d(x, z)+d\left(y, z^{\prime}\right) \geq d(x, T(x, y))+d(y, T(y, x))$, then

$$
\begin{aligned}
& d(x, T(x, y))+d(y, T(y, x)) \\
& \quad \leq\left(1+r+\frac{1}{n}\right)\left(d(x, z)+d\left(y, z^{\prime}\right)\right) .
\end{aligned}
$$

Letting $n \rightarrow \infty$, we have

$d(x, T(x, y))+d(y, T(y, x)) \leq(1+r)\left(d(x, z)+d\left(y, z^{\prime}\right)\right)$.

Thus

$$
\begin{aligned}
\phi(r) & (d(x, T(x, y))+d(y, T(y, x))) \\
& \leq(1-r)(d(x, T(x, y))+d(y, T(y, x))) \\
& \leq \frac{1}{1+r}(d(x, T(x, y))+d(y, T(y, x))) \\
& \leq\left(d(x, z)+d\left(y, z^{\prime}\right)\right),
\end{aligned}
$$

and, from (10), we obtain (44). If $d(x, z)+d\left(y, z^{\prime}\right)<d(x, T(x$, $y))+d(y, T(y, x))$, then

$$
\begin{aligned}
d(x, T & (x, y))+d(y, T(y, x)) \\
\leq & \left(d(x, z)+d\left(y, z^{\prime}\right)\right) \\
& +r\{d(x, T(x, y))+d(y, T(y, x))\} \\
& +\frac{1}{n}\left(d(x, z)+d\left(y, z^{\prime}\right)\right) .
\end{aligned}
$$

And, therefore

$$
\begin{gathered}
(1-r)(d(x, T(x, y))+d(y, T(y, x))) \\
\leq\left(1+\frac{1}{n}\right)\left(d(x, z)+d\left(y, z^{\prime}\right)\right) .
\end{gathered}
$$

Letting $n \rightarrow \infty$, we have

$$
\begin{gathered}
\phi(r)(d(x, T(x, y))+d(y, T(y, x))) \\
\leq\left(d(x, z)+d\left(y, z^{\prime}\right)\right),
\end{gathered}
$$

and, thus from condition (56), we obtain (44).

Finally, from (44), we obtain

$$
\begin{gathered}
d\left(z, T\left(z, z^{\prime}\right)\right)+d\left(z^{\prime}, T\left(z^{\prime}, z\right)\right) \\
=\lim _{n \rightarrow \infty}\left(d\left(x_{n+1}, T\left(z, z^{\prime}\right)\right)+d\left(y_{n+1}, T\left(z^{\prime}, z\right)\right)\right) \\
\leq \lim _{n \rightarrow \infty} r \max \left\{d\left(x_{n}, z\right)+d\left(y_{n}, z^{\prime}\right), d\left(x_{n}, x_{n+1}\right)\right. \\
+d\left(y_{n}, y_{n+1}\right), d\left(z, T\left(z, z^{\prime}\right)\right) \\
+d\left(z^{\prime}, T\left(z^{\prime}, z\right)\right), \\
\frac{d\left(x_{n}, T\left(z, z^{\prime}\right)\right)+d\left(z^{\prime}, T\left(z^{\prime}, z\right)\right)}{2}, \\
\left.\frac{d\left(z, x_{n+1}\right)+d\left(z^{\prime}, y_{n+1}\right)}{2}\right\} \\
\leq r \max \left\{d(z, z)+d\left(z^{\prime}, z^{\prime}\right), d\left(z, T\left(z, z^{\prime}\right)\right)\right. \\
\left.+d\left(z^{\prime}, T\left(z^{\prime}, z\right)\right)\right\} \\
=r d\left(z, T\left(z, z^{\prime}\right)\right)+d\left(z^{\prime}, T\left(z^{\prime}, z\right)\right) .
\end{gathered}
$$

Hence, as $r<1$, we obtain

$$
d\left(z, T\left(z, z^{\prime}\right)\right)+d\left(z^{\prime}, T\left(z^{\prime}, z\right)\right)=0 .
$$

This implies that $z \in T\left(z, z^{\prime}\right)$ and $z^{\prime} \in T\left(z^{\prime}, z\right)$.

Corollary 5. Let $(X, d)$ be a complete metric space and let $T$ be a mapping from $X \times X$ into $\mathrm{CB}(X)$. Assume that there exists $r \in[0,1)$ such that $\phi(r)[d(x, T(x, y))+d(u, T(u, v))] \leq$ $(d(x, u)+d(y, v))$ implies

$$
\begin{aligned}
H(T(x, y), T(u, v)) & \\
\leq \frac{r}{2} \max \{ & d(x, u)+d(y, v), d(x, T(x, y)) \\
& +d(y, T(y, x)), \\
& \quad d(u, T(u, v))+d(v, T(v, u))\}
\end{aligned}
$$

for all $x, y, u, v \in X$, where the function $\phi$ is defined as in Theorem 4. Then, there exist $z, z^{\prime} \in X$ such that $z \in T\left(z, z^{\prime}\right)$ and $z^{\prime} \in T\left(z^{\prime}, z\right)$. 
Corollary 6. Let $(X, d)$ be a complete metric space and let $T$ be a mapping from $X \times X$ into $\mathrm{CB}(X)$. Assume that there exists $r \in[0,1)$ such that $\phi(r)[d(x, T(x, y))+d(u, T(u, v))] \leq$ $(d(x, u)+d(y, v))$ implies

$$
\begin{gathered}
H(T(x, y), T(u, v)) \\
\leq \frac{r}{2} \max \{d(x, u)+d(y, v), d(x, T(x, y)) \\
+d(y, T(y, x)), d(u, T(u, v))+d(v, T(v, u)), \\
\left.\quad \frac{d(u, T(x, y))+d(v, T(y, x))}{2}\right\}
\end{gathered}
$$

for all $x, y, u, v \in X$, where the function $\phi$ is defined as in Theorem 4. Then, there exist $z, z^{\prime} \in X$ such that $z \in T\left(z, z^{\prime}\right)$ and $z^{\prime} \in T\left(z^{\prime}, z\right)$.

\section{An Application}

The existence and uniqueness of solutions of functional equations and system of functional equations arising in dynamic programming have been studied by using various fixed point theorems. In this paper, we shall prove the existence and uniqueness of a solution for a class of functional equations using Corollary 6.

In this section, we assume that $U$ and $V$ are Banach spaces. $W \subset U, D \subset V$, and $\mathfrak{R}$ is a field of real numbers. Let $B(W)$ denote the set of all the real valued functions on $W$. It is known that $B(W)$ endowed with the metric

$$
d_{B}(h, k)=\sup _{x \in W}|h(x)-k(x)|, \quad h, k \in B(W),
$$

is a complete metric space. According to Bellman and Lee, the basic form of the functional equation of dynamic programming is given as

$$
p(x)=\sup _{y} H(x, y, p(\tau(x, y)))
$$

where $x$ and $y$ represent the state and decision vectors, respectively, $\tau: W \times D \rightarrow W$ represents the transformation of the process, and $p(x)$ represents the optimal return function with initial state $x$. In this section, we study the existence and uniqueness of a solution of the following functional equation:

$$
p(x)=\sup _{y}[g(x, y)+G(x, y, p(\tau(x, y)))]
$$

where $g: W \times D \rightarrow \Re$ and $G: W \times D \times \Re \rightarrow \Re$ are bounded functions. In this section, we study the existence and uniqueness of a solution of (59) in the following new form. Let a function $\phi$ be defined as in Theorem 4 and let the mapping $T$ be defined by

$$
\begin{array}{r}
T(h, k)(x) \\
=\sup _{y \in D}[g(x, y)+G(x, y, h(\tau(x, y)), k(\tau(x, y)))], \\
x \in W .
\end{array}
$$

Theorem 7. Suppose that there exists $r \in[0,1)$ such that for every $(x, y) \in W \times D, h, k, h^{\prime}, k^{\prime} \in B(W)$, and $t, s \in W$, the inequality

$$
\begin{gathered}
\phi(r)\left[d_{B}(h, T(h(t), k(s)))+d_{B}\left(h^{\prime}, T\left(h^{\prime}(s), k^{\prime}(t)\right)\right)\right] \\
\leq d\left(h(t), h^{\prime}(s)\right)+d\left(k(s), k^{\prime}(t)\right)
\end{gathered}
$$

implies

$$
\begin{aligned}
& \mid G(x, y, h(\tau(x, y)), k(\tau(x, y))) \\
& -G\left(x, y, h^{\prime}(\tau(x, y)), k^{\prime}(\tau(x, y))\right) \mid \\
& \quad \leq r M\left(h(t), k(s), h^{\prime}(s), k^{\prime}(t)\right),
\end{aligned}
$$

where

$$
\begin{aligned}
M(h(t), & \left.k(s), h^{\prime}(s), k^{\prime}(t)\right) \\
=\max & \left\{\left|h(t)-h^{\prime}(s)\right|+\left|k(s)-k^{\prime}(t)\right|,\right. \\
& |h(t)-T(h(t), k(s))|+|k(s)-T(k(s), h(t))|, \\
& \left|h(s)^{\prime}-T\left(h^{\prime}(s), k^{\prime}(t)\right)\right| \\
& +\left|k^{\prime}(t)-T\left(k^{\prime}(t), h^{\prime}(s)\right)\right|, \\
& \left.\frac{\left|h^{\prime}(s), T(h(t), k(s))\right|+\left|k^{\prime}(t)-T(k(s), h(t))\right|}{2}\right\} .
\end{aligned}
$$

Then, the functional equation (59) has a unique bounded solution in $B(W)$.

Proof. Note that $T$ is a map from $B(W) \times B(W)$ onto $B(W)$ and that $\left(B(W), d_{B}\right)$ is a complete metric space, where $d_{B}$ is the metric defined in (57). Let $\lambda$ be an arbitrary positive real number, and $h, h^{\prime}, k, k^{\prime} \in B(W)$. For arbitrary $x \in W$ and $y \in$ $D$ so that

$$
\begin{aligned}
& T(h, k)(x)<\left[g(x, y)+G\left(x, y, h\left(\tau_{1}\right), k\left(\tau_{2}\right)\right)\right]+\lambda, \\
& T\left(h^{\prime}, k^{\prime}\right)(x)<\left[g(x, y)+G\left(x, y, h^{\prime}\left(\tau_{2}\right), k^{\prime}\left(\tau_{1}\right)\right)\right]+\lambda .
\end{aligned}
$$


From the definition of mapping $T$, we have

$$
\begin{gathered}
T(h, k)(x) \geq\left[g(x, y)+G\left(x, y, h\left(\tau_{2}\right), k\left(\tau_{1}\right)\right)\right], \\
T\left(h^{\prime}, k^{\prime}\right)(x) \geq\left[g(x, y)+G\left(x, y, h^{\prime}\left(\tau_{1}\right), k^{\prime}\left(\tau_{2}\right)\right)\right] .
\end{gathered}
$$

If inequality (61) holds, then from (64) and (67), we get

$$
\begin{aligned}
& T(h, k)(x)-T\left(h^{\prime}, k^{\prime}\right)(x) \\
& \quad<G\left(x, y, h\left(\tau_{1}\right), k\left(\tau_{2}\right)\right)-G\left(x, y, h^{\prime}\left(\tau_{1}\right), k^{\prime}\left(\tau_{2}\right)\right)+\lambda \\
& \quad \leq\left|G\left(x, y, h\left(\tau_{1}\right), k\left(\tau_{2}\right)\right)-G\left(x, y, h^{\prime}\left(\tau_{1}\right), k^{\prime}\left(\tau_{2}\right)\right)\right|+\lambda \\
& \quad \leq r M\left(h(t), k(s), h^{\prime}(s), k^{\prime}(t)\right)+\lambda .
\end{aligned}
$$

Similarly, (65) and (66) implies that

$$
\begin{aligned}
& T\left(h^{\prime}, k^{\prime}\right)(x)-T(h, k)(x) \\
& \quad \leq r M\left(h(t), k(s), h^{\prime}(s), k^{\prime}(t)\right)+\lambda .
\end{aligned}
$$

Hence, from (68) and (69), we have

$$
\begin{aligned}
& \left|T(h, k)(x)-T\left(h^{\prime}, k^{\prime}\right)(x)\right| \\
& \quad \leq r M\left(h(t), k(s), h^{\prime}(s), k^{\prime}(t)\right)+\lambda .
\end{aligned}
$$

Since inequality (70) is true for any $x \in W$ and arbitrary $\lambda>$ 0 , then (61) implies

$$
\begin{aligned}
d_{B}\left(T(h(t), k(s)), T\left(h^{\prime}(s), k^{\prime}(t)\right)\right) & \\
\leq r \max & \left\{\left|h(t)-h^{\prime}(s)\right|+\left|k(s)-k^{\prime}(t)\right|,\right. \\
& |h(t)-T(h(t), k(s))| \\
+ & |k(s)-T(k(s), h(t))|, \\
& \left|h(s)^{\prime}-T\left(h^{\prime}(s), k^{\prime}(t)\right)\right| \\
+ & \left|k^{\prime}(t)-T\left(k^{\prime}(t), h^{\prime}(s)\right)\right|, \\
& \left.\frac{\left|h^{\prime}(s), T(h(t), k(s))\right|+\left|k^{\prime}(t)-T(k(s), h(t))\right|}{2}\right\} .
\end{aligned}
$$

Therefore, all the conditions of Corollary 6 are met for the mapping $T$, and hence the functional equation (59) has a unique bounded solution.

\section{Conflict of Interests}

The authors declare that there is no conflict of interests regarding the publication of this paper.

\section{References}

[1] T. Suzuki, "A generalized Banach contraction principle that characterizes metric completeness," Proceedings of the American Mathematical Society, vol. 136, no. 5, pp. 1861-1869, 2008.

[2] S. Banach, "Sur les operations dans les ensembles abstraits et leur application aux equations integrales," Fundamenta Mathematicae, vol. 3, pp. 133-181, 1922.

[3] M. Kikkawa and T. Suzuki, "Some similarity between contractions and Kannan mappings," Fixed Point Theory and Applications, vol. 2008, Article ID 649749, 8 pages, 2008.

[4] R. Kannan, "Some results on fixed points. II," The American Mathematical Monthly, vol. 76, pp. 405-408, 1969.

[5] S. B. Nadler Jr., "Multi-valued contraction mappings," Pacific Journal of Mathematics, vol. 30, pp. 475-488, 1969.

[6] N. Mizoguchi and W. Takahashi, "Fixed point theorems for multivalued mappings on complete metric spaces," Journal of Mathematical Analysis and Applications, vol. 141, no. 1, pp. 177188, 1989.

[7] P. Z. Daffer and H. Kaneko, "Fixed points of generalized contractive multi-valued mappings," Journal of Mathematical Analysis and Applications, vol. 192, no. 2, pp. 655-666, 1995.

[8] P. V. Semenov, "On fixed points of multivalued contractions," Functional Analysis and Its Applications, vol. 36, no. 2, pp. 159$161,2002$.

[9] L. Ćirić, "Multi-valued nonlinear contraction mappings," Nonlinear Analysis. Theory, Methods \& Applications, vol. 71, no. 7-8, pp. 2716-2723, 2009.

[10] L. B. Ćirić, "Fixed points for generalized multi-valued contractions," Matematički Vesnik, vol. 9, no. 24, pp. 265-272, 1972.

[11] D. Doric and R. Lazović, "Some Suzuki-type fixed point theorems for generalized multivalued mappings and applications," Fixed Point Theory and Applications, vol. 2011, article 40, 2011.

[12] T. Gnana Bhaskar and V. Lakshmikantham, "Fixed point theorems in partially ordered metric spaces and applications," Nonlinear Analysis. Theory, Methods \& Applications, vol. 65, no. 7, pp. 1379-1393, 2006. 


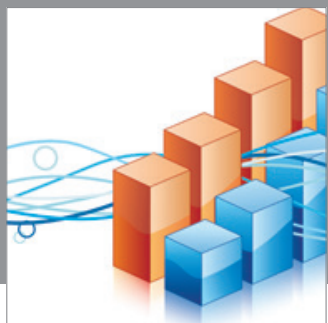

Advances in

Operations Research

mansans

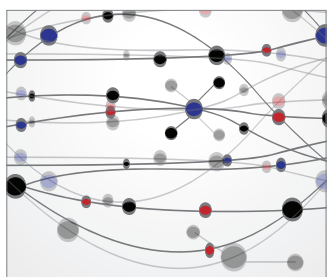

The Scientific World Journal
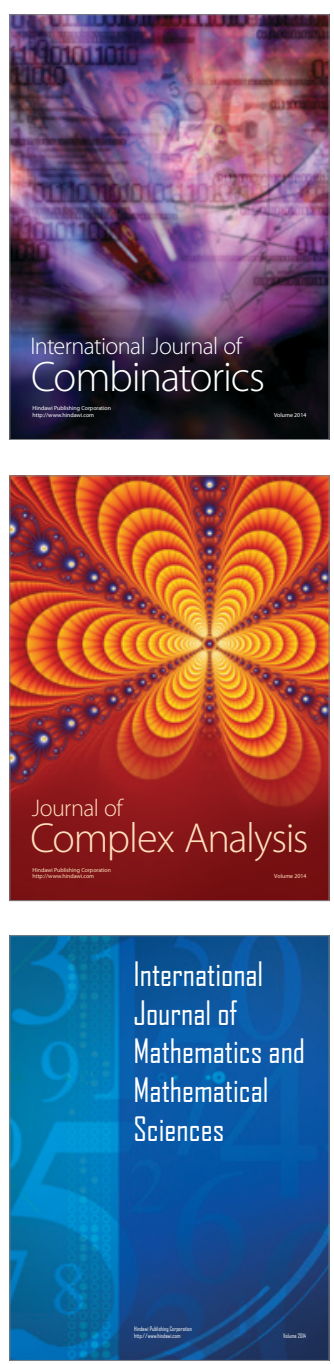
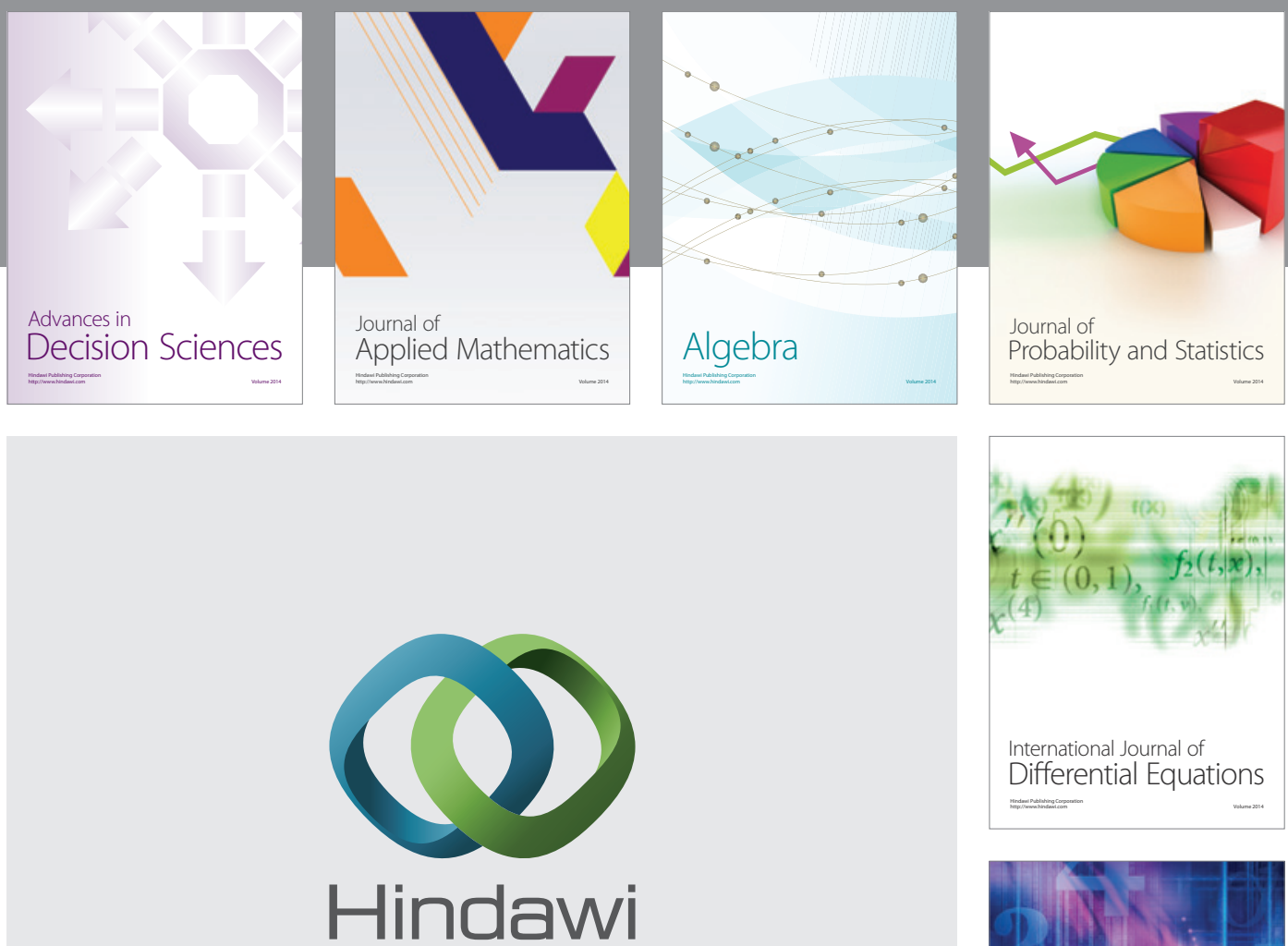

Submit your manuscripts at http://www.hindawi.com
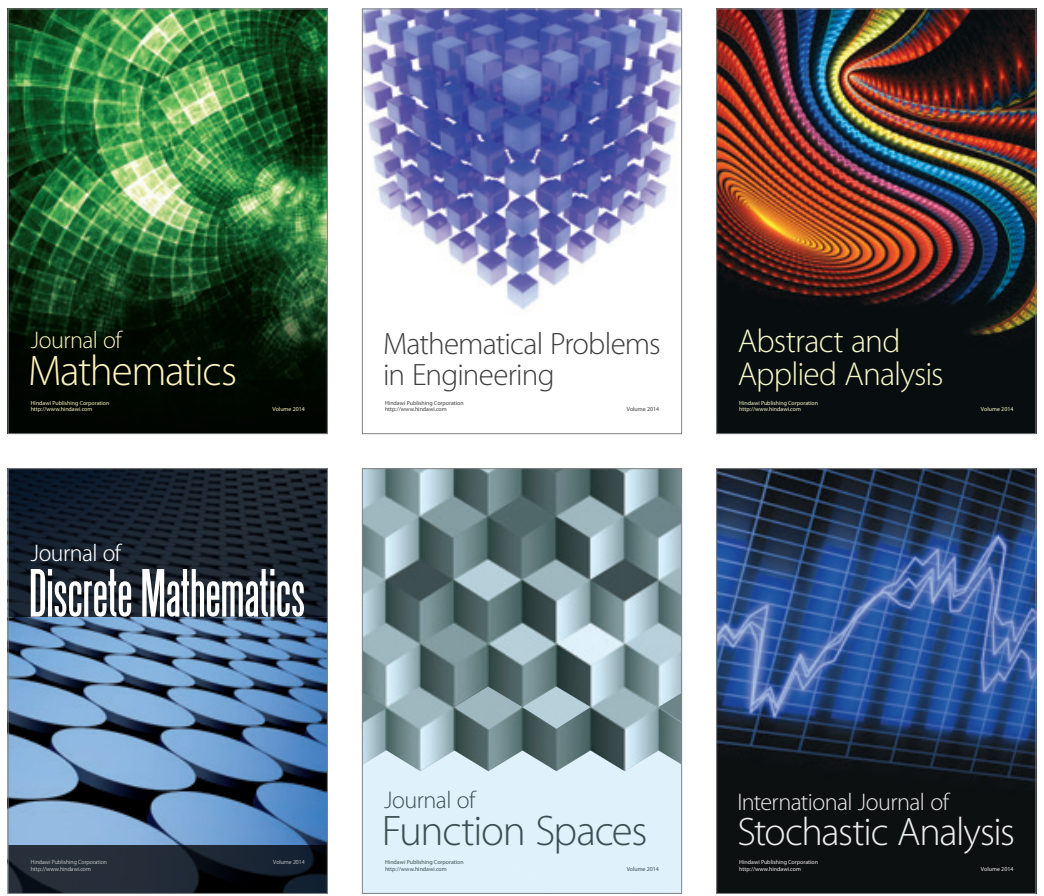

Journal of

Function Spaces

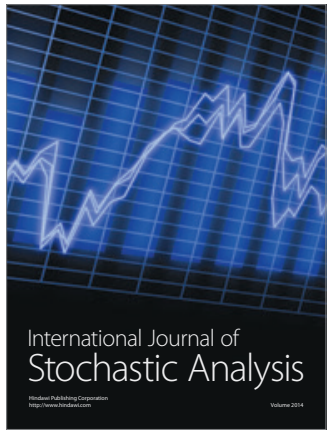

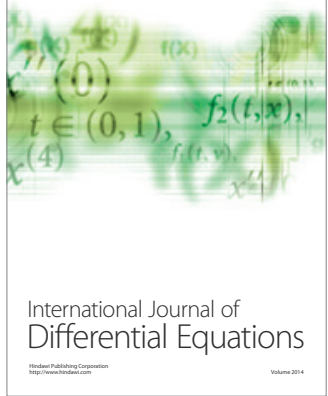
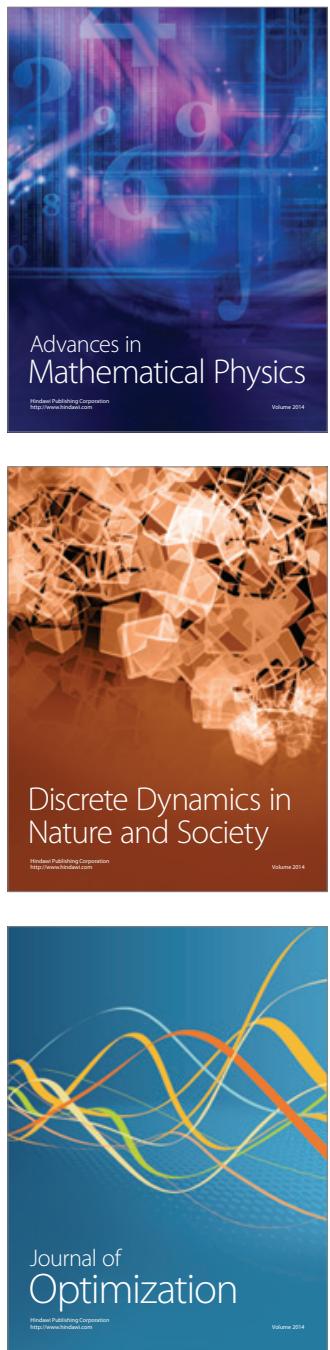